\title{
Genetic Variation in the Carbonic Anhydrase Isozymes of Macaque Monkeys. II. Inheritance of Red Cell Carbonic Anhydrase Levels in Different Carbonic Anhydrase I Genotypes of the Pig-Tailed Macaque, Macaca nemestrina ${ }^{1}$
}

\author{
Joseph DeSimone, ${ }^{2,3}$ Erik Magid, ${ }^{2,4}$ and Richard E. Tashian ${ }^{2}$
}

Received 21 Feb. 1972-Final 8 May 1972

The inheritance of red blood cell levels of carbonic anhydrase isozymes (CA I and $C A I I$ ) has been studied in different carbonic anhydrase I genotypes of the pig-tailed macaque, Macaca nemestrina. Quantitation of $C A I$ isozymes in a series of animals indicates that the total $C A$ I concentration is the sum of the average effects of each $C A I$ structural allele and that the average effects are independent of the various allelic combinations. The relative average effects were 0.32:0.95:1.0 for the $\mathrm{CA} \mathrm{I}^{\mathrm{a}}, \mathrm{CA} \mathrm{I}^{\mathrm{b}}$, and $\mathrm{CA} \mathrm{I}^{\mathrm{c}}$ structural genes, respectively. It is also demonstrated that the level of $C A I I$ is related to the $C A I$ genotypes. Multiple regression analysis demonstrated that each dose of $C A$ $I$-deficiency gene present decreased the CA II concentration by approximately $30 \%$, with this decrease in $C A$ II level being solely related to the dose of $C A$ I-deficiency gene and not to the level of CA I. The CA I-deficient animals produce $C A I$ products that are similar to the common $C A I a, C A I b, C A I c$ electrophoretic types. Limited mating data indicate that the $C A I$ components in CAI-deficient animals are inherited codominantly.

\footnotetext{
Supported by U.S. Public Health Service Research Grant GM-15419.

${ }^{1}$ This report is a portion of a dissertation submitted to the University of Michigan in partial fulfillment of the requirements for the Doctor of Philosophy degree.

2 Department of Human Genetics, University of Michigan Medical School, Ann Arbor, Michigan.

${ }^{3}$ U.S. Public Health Service Predoctoral Trainee (GM-71-14).

${ }^{4}$ U.S. Public Health Service International Postdoctoral Fellow. Present address: Department of Clinical Chemistry, Bispebjerg Hospital, Bispebjerg Bakke 23, 2400 Copenhagen NV, Denmark.
} 


\section{INTRODUCTION}

Extensive electrophoretic and quantitative variation for the two isozymes of erythrocyte carbonic anhydrase has been reported for Macaca nemestrina (Headings and Tashian, 1971; Tashian et al., 1971).

In the preceding paper (Magid et al., 1973), it was demonstrated that a quantitative relationship exists between the electrophoretic types of CA I and the levels of that isozyme in the erythrocytes and also that there is an association between the concentrations of CA I and CA II.

In this paper, the carbonic anhydrase system in $M$. nemestrina is further characterized, with particular reference to (1) the correlation between CA I concentration and CA I genotypes, (2) the correlation between CA I and CA II concentrations, and (3) the inheritance of CA I electrophoretic types in CA I-deficient homozygotes.

\section{Genetic Nomenclature}

The structural alleles at the $C A I$ locus will be designated $I^{a}, I^{b}, I^{c}$, and $I^{d}$, and the structural alleles at the $C A I I$ locus will be designated $I I^{a_{2}}$ and $I I^{b}$. The CA I-deficiency gene and the normal allele at this locus will be designated $X^{-}$and $X^{+}$, respectively. For example, an animal that is phenotypically CA Iab will have the genotype $X^{+} I^{a} / X^{+} I^{b}$. It will be demonstrated later in this paper that CA I-deficient animals can have CA I electrophoretic types that are apparently identical to the common CA I electrophoretic types. Therefore, CA I-deficient animals whose CA I electrophoretic types are known will have the following genotypes:

$$
X^{-} I^{a} / X^{-} I^{a}, X^{-} I^{a} / X^{-} I^{b}, \text { etc. }
$$

A suppressed CA I structural gene whose CA I electrophoretic type is unknown will be designated $X^{-} I^{x}$.

\section{EXPERIMENTAL PROCEDURES}

\section{Materials}

Whole blood samples were obtained from a breeding colony of $M$. nemestrina at the Washington Regional Primate Center, Medical Lake, Washington.

\section{Quantitation of Carbonic Anhydrase Isozymes}

The concentrations of each carbonic anhydrase form (CA I and CA II) were determined in crude hemolysates with a radioimmunosorbent assay (Magid et al., 1973). CA I phenotypes were determined using vertical starch gel electrophoresis as previously described (Tashian et al., 1971). 


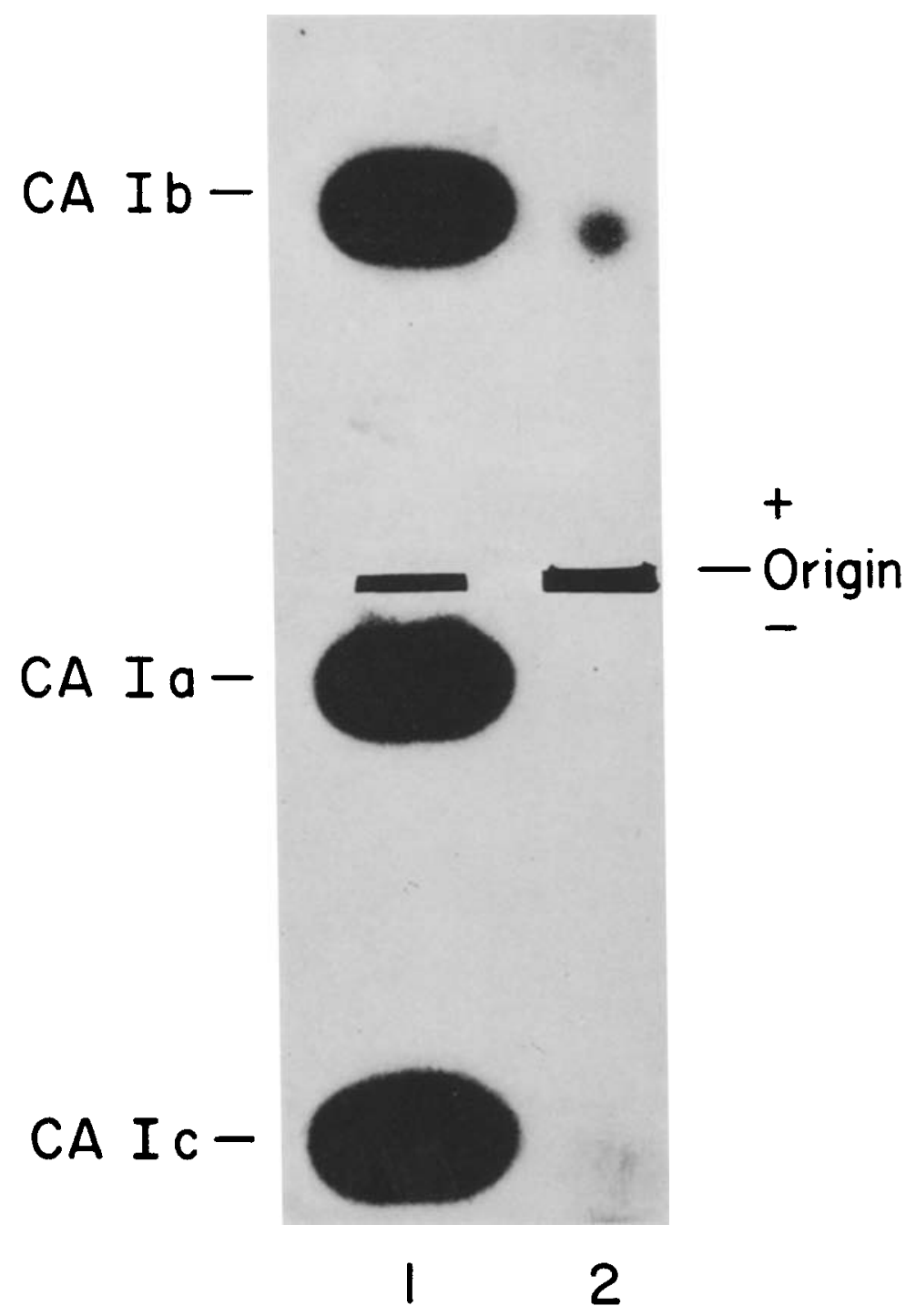

Fig. 1. Starch gel electrophoresis of (1) a marker sample made up of equal concentrations of purified CA Ia, CA Ib, and CA Ic and (2) a concentrated chloroform-ethanol extract of a CA I-deficient hemolysate. The CA I-deficient pattern exhibits esterase staining in the $\mathrm{CA} \mathrm{Ib}$ and $\mathrm{CA} \mathrm{Ic}$ regions of the starch gel. The material in these regions cross-reacts with anti-CA I serum. 


\section{Multiple Regression Analysis}

Multiple regression analysis was performed with the aid of a University of Michigan program (BMDO3R) written for the IBM 360/67 computer. The program was written by the Health Sciences Computing Facility, University of California, Los Angeles.

\section{Detection of CA I Electrophoretic Types in CA I-Deficient Animals}

The present technique couples electrophoresis in starch gel with product analysis by the radioimmunosorbent assay (Magid et al., 1973). Twomilliliter samples of hemolysate from CA I-deficient animals were extracted in chloroform and ethanol (Tashian et al., 1966), and the precipitated hemoglobin was removed by centrifugation $(10,000 \times g$ for $30 \mathrm{~min}$ at $4 \mathrm{C})$. The supernatant was then concentrated by lyophilization, and this powder was redissolved in $0.2 \mathrm{ml}$ of distilled water. By this procedure, a five- to sevenfold concentration of carbonic anhydrase was realized. The concentrated enzyme solutions were then subjected to vertical starch gel electrophoresis (Tashian, 1969), and the regions corresponding to $\mathrm{CA} \mathrm{Ia,} \mathrm{Ib}$, and Ic were cut from the gel with the aid of a marker sample containing all three electrophoretic types (see Fig. 1). Each region of starch gel was subsequently homogenized, and th eCA I was eluted into $2 \mathrm{ml}$ of assay buffer (Wide, 1969) and analyzed for CA I cross-reactivity by the radioimmunosorbent assay. This method uses the radioassay qualitatively; any region that inhibits the binding of $\mathrm{I}^{125}$-labeled antigen is considered to contain CA I cross-reacting material. Approximately $10 \%$ of a purified CA Ia preparation added to the starch gel at a concentration of $3 \mu \mathrm{g} / \mathrm{ml}$ was recovered in the eluate from the CA Ia region of the starch gel.

\section{RESULTS AND DISCUSSION}

\section{Quantitation of Carbonic Anhydrase Isozymes}

CA I and CA II isozyme concentrations were recently determined in animals with different CA I phenotypes (Magid et al., 1973). Results of these quantitations indicated a relationship between the CA I phenotype of an animal and its CA I concentration, and also indicated an association between CA I and CA II concentrations. The concentrations of the CA I and CA II isozymes have been determined for an additional series of animals. The results of both studies were combined and are presented in Table I.

An attempt has been made in Table I to group animals with respect to their assumed CA I genotypes, rather than to their CA I phenotypes. This has been done to preserve as much genetic information as possible. Because 
Table I. CA I and CA II Concentrations Associated with Each CA I Genotype

\begin{tabular}{lccc}
\hline \multicolumn{1}{c}{$\begin{array}{c}\text { Proposed } \\
\text { CA I genotype }\end{array}$} & $\begin{array}{c}\text { CA I } \\
\text { concentration } \\
(\mu \mathrm{g} / \mathrm{mg} \mathrm{Hb})\end{array}$ & & $\begin{array}{c}\text { CA II } \\
\text { concentration } \\
(\mu \mathrm{g} / \mathrm{mg} \mathrm{Hb})\end{array}$ \\
& $\overline{\mathbf{x}} \pm \mathrm{SD}$ & & $\overline{\mathbf{x}} \pm \mathrm{SD}$ \\
\hline$X^{-} I^{x} / X^{-} I^{x}(16)^{a}$ & $0.002 \pm 0.002$ & & $1.07 \pm 0.16$ \\
$X^{-} I^{x} / X^{+} I^{a}(19)$ & $2.71 \pm 0.68$ & & $1.92 \pm 0.31$ \\
$X^{+} I^{a} / X^{+} I^{a}(3)$ & 6.57 & & 2.25 \\
$X^{-} I^{x} / X^{+} I^{b}(21)$ & $8.09 \pm 1.28$ & & $1.89 \pm 0.32$ \\
$X^{+} I^{a} / X^{+} I^{b}(17)$ & $11.02 \pm 1.95$ & & $2.64 \pm 0.35$ \\
$X^{+} I^{a} / X^{+} I^{c}(5)$ & $11.54 \pm 0.88$ & & $2.47 \pm 0.22$ \\
$X^{+} I^{b} / X^{+} I^{c}(2)$ & 18.50 & & 2.25 \\
\hline
\end{tabular}

${ }^{a}$ Number of animals tested.

of the small family size and long generation time of primates, it is very difficult to identify the genotypes of all animals with certainty. Animals that are heterozygous for different electrophoretic types (i.e., $X^{+} I^{a} / X^{+} I^{b}$, $\left.X^{+} I^{a} / X^{+} I^{c}, X^{+} I^{b} / X^{+} I^{c}\right)$ or those homozygous for the deficiency gene $X^{-} I^{x} / X^{-} I^{x}$, can be classified from starch gel patterns. Those animals whose phenotype is CA Ia or CA Ib (only one animal with the CA Ic phenotype was found in the mating population) may or may not be heterozygous for the deficiency gene. In most instances, heterozygotes in these classes can be determined using the available mating data. For example, in the case where a CA Ia male is mated to a CA Iab female to produce a $\mathrm{CA} \mathrm{Ib}$ offspring, it is obvious that the genotype of the male parent is $X^{-} I^{x} / X^{+} I^{a}$ and that of the offspring is $X^{-} I^{x} / X^{+} I^{b}$. The only critical matings that can be used to determine homozygosity for CA Ia or CA Ib animals would be those matings of $\mathrm{CA}$ Iab parents to produce CA Ia or CA Ib offspring. The genotypes of these offspring would therefore be $X^{+} I^{a} / X^{+} I^{a}$ or $X^{+} I^{b} / X^{+} I^{b}$, respectively. Unfortunately, matings of this type are rare. However, if all animals of phenotype Ia or Ib are classified as genotypically $X^{-} I^{x} / X^{+} I^{a}$ or $X^{-} I^{x} / X^{+} I^{b}$, respectively, the error of misclassification would be small, since only $15 \%$ of all phenotypically $\mathrm{Ia}$ or $\mathrm{Ib}$ animals (on the basis of calculations from population gene frequencies) are expected to be $X^{+} I^{a} / X^{+} I^{a}$ or $X^{+} I^{b} / X^{+} I^{b}$.

It is obvious from Table I that CA I concentration is correlated with the CA I genotype $(r=0.94)$. Closer inspection reveals the total CA I concentration to be the sum of the average effects of each allele and the average effects to be independent of the various allelic combinations. Determinations of the concentration of CA I electrophoretic types in CA Iab heterozygotes have also been made using quantitative cellulose acetate electrophoresis (Ruis-Reys and Ramirez-Zorilla, 1968) or column chromatography on 
DEAE A50 (Tanis et al., 1970). The concentrations of CA Ia and Ib in these heterozygotes are the same as the predicted average effects from Table I.

Similar findings have been reported for human red cell acid phosphatases (Spencer et al., 1964). Three acid phosphatase alleles $\left(P^{a}, P^{b}\right.$, and $P^{c}$ ) were studied in a randomly selected group of English adults. The relative contribution of each allele to the total acid phosphatase activity was found to be 2:3:4 for $P^{a}, P^{b}$, and $P^{c}$, respectively. Whether the activity differences were related to protein concentration differences or to specific activity differences was not determined at that time. Acid phosphatases were later partially purified from the red cells of homozygous $P^{a} / P^{a}$ and $P^{b} / P^{b}$ individuals (Scott 1966). Kinetic properties of these two enzymes differ slightly, but the differences do not appear sufficient to explain the marked difference in total activity observed in human red cells.

The cause of the concentration difference between CA Ia and CA Ib has not been determined. Evidence to be presented elsewhere (DeSimone et al., 1973b) indicates that this difference is not related to different rates of synthesis.

\section{Association Between CA I Genotypes and the Concentration of CA I and CA II}

CA II concentration appears to be associated with either the CA I genotype or the CA I concentration, or with both of these factors (Table I). To partition the association, a multiple linear regression analysis was performed with CA II concentration as the dependent variable. The CA I genotype, the CA I concentration, and the interaction term for CA I genotype and CA I concentration were the independent variables. Partial regression coefficients, adjusted for CA I concentration, were computed for two scales of CA I genotype. One scale ranks CA I genotype with respect to the dose of nondeficiency genes present and therefore focuses on the effect of the deficiency gene. The other classification takes advantage of information available from each of the seven CA I genotypes. Values from 1 through 7 were assigned for a direct ranking of the average concentration of CA I for each CA I genotype.

Results of the analysis using the dose of nondeficiency genes for ranking CA I genotype are presented in Table II. The only significant partial regression coefficient is that due to the dose of nondeficiency genes $(P<0.01)$. Although the CA I concentration is correlated with the dose of nondeficiency gene (simple correlation coefficient 0.83 ), the CA I concentration, adjusted for CA I genotype differences, does not appear to be a factor in determining the concentration of CA II. When the analysis was performed using all seven genotype classes, no changes in the regression were observed.

Table III lists the CA II concentrations associated with each dose of the 
Table II. Multiple Regression Analysis: Dependent Variable, [CA II]; Independent Variables, [CA I], CA I Genotype Value, and [CA I] $\times$ CA I Genotype Value ${ }^{a}$

\begin{tabular}{lcrc}
\hline \multicolumn{1}{c}{ Variable } & $\begin{array}{c}\text { Partial } \\
\text { regression coefficient }\end{array}$ & $F$ value & Significance \\
\hline [CA I] & 0.038 & 1.73 & N.S. \\
CA I genotype value & 0.734 & 61.63 & P $<0.01$ \\
[CA I] $\times$ CA I genotype value & 0.013 & 1.50 & N.S. \\
& & & \\
\hline
\end{tabular}

${ }^{a}$ Multiple regression equation: $[\mathrm{CA} \mathrm{II}]=k+b_{1} .[\mathrm{CA} \mathrm{I}]+b_{.2} \times(\mathrm{CA}$ I genotype value $)$ $+b_{12}[\mathrm{CA}$ I] $\times$ (CA I genotype value).

Table III. CA II Concentrations Associated with Dosage of CA IDeficiency Gene

\begin{tabular}{lcc}
\hline \multirow{2}{*}{$\begin{array}{c}\text { CA I } \\
\text { genotype }\end{array}$} & $\begin{array}{c}\text { CA II } \\
\text { concentration } \\
(\mu \mathrm{g} / \mathrm{mg} \mathrm{Hb})\end{array}$ & $\begin{array}{c}\text { Conditional } \\
\text { correlation coefficient }\end{array}$ \\
\cline { 2 - 3 } & $\bar{x} \pm \mathrm{SD}$ & \\
\hline$X^{-} I^{x} / X^{-} I^{x}(16)^{a}$ & $1.07 \pm 0.16$ & -0.02 \\
$X^{-} I^{x} / X^{+} I^{\nu b}(40)$ & $1.90 \pm 0.31$ & 0.040 \\
$X^{+} I^{y} / X^{+} I^{y}(27)$ & $2.53 \pm 0.33$ & 0.080 \\
\hline
\end{tabular}

${ }^{a}$ Number of animals tested.

${ }^{b} y$ is a general designation for a nonsuppressed CA I structural allele.

normal $\left(X^{+}\right)$gene. Also included are the conditional correlation coefficients of CA I concentration on CA II concentration, given the dose of the $X^{+}$ gene. Each dose of deficiency gene $\left(X^{-}\right)$reduces the CA II concentration by approximately $30 \%$, with the effect of dose being additive. The fact that the conditional correlation coefficients are close to zero also indicates that the CA I concentration is probably not a determining factor in the regulation of CA II levels.

\section{Inheritance of Electrophoretically Distinguishable CA I Products in CA I-Deficient Homozygotes}

By concentrating large volumes of blood (Headings and Tashian, 1971), it was possible to demonstrate that a small amount of CA I is synthesized in homozygous CA I-deficient animals. A component was distinguishable on starch gel electrophoresis that (1) migrates in the position of CA Ia, (2) has a similar enzymatic activity for $\beta$-naphthyl acetate, (3) is inhibited by Diamox, a specific inhibitor of carbonic anhydrase, and (4) is immunologically identical to purified CA $\mathrm{I}$ on immunodiffusion. In all bloods 
Table IV. Inheritance of CA I Electrophoretic Types in CA I-Deficient Homozygotes

\begin{tabular}{ccccc}
\hline $\begin{array}{c}\text { Proposed genotype } \\
\text { of } \\
\text { male parent }\end{array}$ & & $\begin{array}{c}\text { Proposed genotypes } \\
\text { of } \\
\text { female parent }\end{array}$ & & $\begin{array}{c}\text { Proposed genotypes } \\
\text { of } \\
\text { offspring }\end{array}$ \\
\hline$X^{-} I^{a} / X^{-} I^{c}$ & $\times$ & $X^{-} I^{b} / X^{-} I^{c}$ & $\rightarrow$ & $X^{-} I^{a} / X^{-} I^{b}$ \\
& $\times$ & $X^{-} I^{b} / X^{-} I^{c}$ & $\rightarrow$ & $X^{-} I^{a} / X^{-} I^{b}$ \\
& $\times$ & $X^{-} I^{b} / X^{-} I^{c}$ & $\rightarrow$ & $X^{-} I^{b} / X^{-} I^{c}$ \\
\hline
\end{tabular}

a The same male was mated to the three females listed in the second column.

examined, both from pooled samples and single individuals, only a component which could be classified as CA Ia was found.

To determine whether the CA I product in CA I-deficient animals is associated only with the CA Ia electrophoretic type, an additional series of animals was typed by the concentration procedure of Headings and Tashian (1971). A sufficient quantity of blood was available from each of five additional CA I-deficient animals to permit typing. In this small sample of animals, the $\mathrm{CA} \mathrm{Ia,} \mathrm{CA} \mathrm{Ib}$, and CA Ic electrophoretic types were present. In addition, a hemolysate was identified with esterase activity in both the $\mathrm{CA} \mathrm{Ib}$ and CA Ic regions of the gel (Fig. 1). This indicates that some CA I-deficient animals may also be heterozygous for CA I electrophoretic types. It seems likely, therefore, that the mutation responsible for the CA I deficiency has occurred at a site which is distinct from those responsible for the electrophoretic variants of CA I.

Because it is difficult to obtain large volumes of blood from single animals, a sensitive immunological procedure, described above, was used for additional typing of CA I-deficient animals. The results of the typing of a small series of animals by this procedure demonstrated the presence, in hemolysates, of CA Ia, CA Ib, and CA Ic electrophoretic types.

During the process of screening the breeding colony at the Washington Regional Primate Center for CA I and CA II variants, it was found that three matings were available that might possibly give some information regarding the inheritance of the CA I electrophoretic types in CA I-deficient animals. The mating data presented in Table IV, although based on only three crosses, are nevertheless compatible with a codominant mode of inheritance.

\section{Possible Genetic Control Mechanisms}

The results of experiments relating to the quantitative control of the carbonic anhydrases in $M$. nemestrina red blood cells have revealed a complex system 
suggesting many possible control mechanisms. It has previously been suggested (Tashian et al., 1971) that the CA I-deficiency mutation acts only in the cis position and has the effect of either suppressing the synthesis of CA I or interfering in some manner with the formation or stability of CA I. Since no incorporation of $\mathrm{C}^{14}$-L-serine into $\mathrm{CA} I$ from reticulocytes of CA I-deficient animals could be demonstrated (DeSimone et al., 1973b), it is possible that (1) CA I may be preferentially lost as newly forming or newly formed molecules, or (2) CA I is synthesized at a 5000-fold reduced rate. The CA I-deficiency mutation affects the concentration of at least three of the CA I electrophoretic types but does not measurably change their catalytic, electrophoretic, or immunological properties. It seems unlikely, therefore, that the CA I-deficiency mutation could cause a gross structural change which renders the CA I molecule to be almost completely unstable and at the same time does not change any of its other chemical properties.

The reduction of CA II concentration associated with the CA Ideficiency phenotype has been shown to result from a decreased rate of synthesis rather than an increased rate of degradation (DeSimone et al., 1973b). This observation, together with the finding of a dosage effect of the CA-I deficiency gene on CA II concentration, may have some bearing on the nature of the CA I-deficiency mutation. Since the decreased rate of synthesis of CA II is not related to any feedback mechanism by CA I protein, it may be assumed that the CA I-deficiency mutation exerts its effect through either its mRNA or its DNA. The possibility that reduced synthesis of CA II is related to a control element shared by both CA I and CA II is unlikely, because no trans effect of the CA I-deficiency mutation is observed on CA I synthesis by the homologous chromosome.

Carbonic anhydrase isozymes are probably the products of gene duplication ( $c f$. Tashian et al., 1972), and their structural genes appear to be closely linked (DeSimone et al., 1973a). As such, the CA I-deficiency mutation could exert a polar effect on CA II synthesis.

\section{ACKNOWLEDGMENTS}

We are grateful to Dr. Fredric D. Abramson, Dr. Charles F. Sing, and Dr. Richard H. Ward for their helpful suggestions during the course of this study. We would like to thank Dr. Orville A. Smith, Dr. Gerald Blakley, Dr. William Morton, Mrs. Dale Hadden, and their colleagues at the University of Washington Regional Primate Research Center for their prompt and generous help in securing the blood samples used in this study. The skillful research assistance of Mrs. Ya-Shiou L. Yu is also gratefully acknowledged. 


\section{REFERENCES}

DeSimone, J., Linde, M., and Tashian, R. E. (1973a). Evidence for linkage of carbonic anhydrase isozyme genes in the pig-tailed macaque, Macaca nemestrina. Nature New Biol. (in press).

DeSimone, J., Magid, E., and Tashian, R. E. (1973b). Genetic variation in the carbonic anhydrase isozymes of macaque monkeys. III. In vitro synthesis of carbonic anhydrases in the reticulocytes of the pig-tailed macaque, Macaca nemestrina. Submitted for publication.

Headings, V. E., and Tashian, R. E. (1971). Quantitative genetic variation in carbonic anhydrase isozymes from tissues of the pig-tailed macaque, Macaca nemestrina. Biochem. Genet. 5:333.

Magid, E., DeSimone, J., and Tashian, R. E. (1973). Genetic variation in the carbonic anhydrase isozymes of macaque monkeys. I. Radioimmunosorbent assay. Biochem. Genet. 8:157.

Ruis-Reys, G., and Ramirez-Zorilla, M. J. (1968). Rapid measurement of erythrocyte carbonic anhydrase isozymes by means of cellulose acetate membrane electrophoresis. Am. J. Clin. Pathol. 50:677.

Scott, E. M. (1966). Kinetic comparison of genetically different acid phosphatases of human erythrocytes. J. Biol. Chem. $241: 3049$.

Spencer, N., Hopkinson, D. A., and Harris, H. (1964). Quantitative differences and gene dosage in the human red cell acid phosphatase polymorphism. Nature 201:299.

Tanis, R. J., Tashian, R. E., and Yu, Y. L. (1970). Properties of carbonic anhydrase isozymes isolated from porcine erythrocytes. J. Biol. Chem. 245:6003.

Tashian, R. E. (1969). The esterases and carbonic anhydrases of human erythrocytes. In Yunis, J. J. (ed.), Biochemical Methods in Red Cell Genetics, Academic Press, New York, p. 307.

Tashian, R. E., Riggs, S. K., and Yu, Y.-S. L. (1966). Characterization of a mutant human erythrocyte carbonic anhydrase: Carbonic anhydrase Ic Guam. Arch. Biochem. Biophys. 117:320.

Tashian, R. E., Goodman, M., Headings, V. E., DeSimone, J., and Ward, R. H. (1971). Genetic variation and evolution in the red cell carbonic anhydrase isozymes of macaque monkeys. Biochem. Genet. 5:183.

Tashian, R. E., Tanis, R. J., and Ferrell, R. E. (1972). Comparative aspects of the primary structures and activities of mammalian carbonic anhydrases. Alfred Benzon Symp. 4:399.

Wide, L. (1969). Radioimmunoassays employing immunosorbents. Acta Endocrinol. 63:207 (suppl. 142). 